\title{
W. B. Herrmannsfeldt
}

Stanford Linear Accelerator Center

Stanford University, Stanford, California 94305

\section{Introduction}

This paper is a status report on the development of the Recirculating Linear Accelerator (RLA) at the Stanford Linear Accelerator Center. The RLA is the third and least expensive method of substantially increasing the energy of the two-mile linear accelerator that has been studied. The original concept of SLAC contemplated a later "phase II" in which the present complement of 240-high power klystrons would be quadrupled, approximately doubling the peak beam energy capability. The estimated cost of the full phase II was approximately $\$ 80$ millicn, while less comprehensive stages would have proportionately lower costs. The second method studied was to replace the present copper accelerator structure with a superconducting microwave structure made of niobium at an estimated cost of $\$ 100$ million. This expense seemed justified only if the final beam energy of the accelerator could be in the $100 \mathrm{GeV}$ range for which peak field gradients in the range of $10 \mathrm{MeV} /$ foot would be required. Such fields have proven most difficult to achieve even in laboratory tests and practical production of such a superconducting structure appears clearly beyond the present state of the art.

Encouraged by the productivity of the physics results from SLAC, and seeking a more immediate plan to increase the energy capability of the accelerator, the Laboratory last year proposed to construct a Recirculating Linear Accelerator. This device, which would double the peak energy of the accelerator, was estimated to cost about $\$ 18$ million and take $2 \frac{1}{2}$ years to construct. Also last year, a Task Force was created under the leadership of John Rees to further develop the plans for the project in anticipation of federal authorization in Fiscal Year 1974. The RLA was not included in the Federal Budget for FY '74, but the Laboratory plans to resubmit the proposal for FY 75 . Therefore studies on the RIA are continuing and the current status of these studies are the substance of the rest of this report.

\section{General Description}

The principle of the Recirculating Linear Accelerator (RLA) is illustrated in Fig. 1. A new beam from the injector is accelerated once through the existing linear accelerator to an energy of $20 \mathrm{GeV}$ or less. This beam is then extracted from the accelerator and inflected into the $6.9 \mathrm{~km}$ long recirculator for 120 revolutions corresponding to che machine interpulse period of the linac $(1 / 360 \mathrm{~s}$ or $2.8 \mathrm{~ms})$. Then, when the modulators have recharged and the klystron system is ready for the next RF pulse, the stored beam is extracted from the recirculator and reinserted into the linear accelerator for a $s^{*}$ cond pass. The maximum beam energy is thus increased to $\sim 45 \mathrm{GeV}$ by the second acceleration. Alternately, it is possibie to use the recirculator as a "beam stretcher"; every time the $1.6 \mu \mathrm{s}$ train of electrons approaches the east loop, a fraction of the beam is spilled into the beam switchyard, which conducts it to the experimental areas. Since the recirculating period is $23 \mu \mathrm{s}$ and the beam pulse is $1.6 \mu \mathrm{s}$ long, the resulting duty cycle is $7 \%$.

Both the low-energy beam from the injector and the highenergy recirculated beam can be contained simultaneously in the two-mile accelerator so that the full repetition rate of 360 pulses per second can be retained in RLA. In this mode of operation, the reinjected high-energy beam bunches will merge with the newly injected first-pass bunches on the

*Work supported by the U. S. Atomic Energy Commission. crests of the accelerating wave and pass together down the linac.

The recirculator proper consists of two $95-\mathrm{m}$-radius loops located at the ends of the 3-km-long accelerator and joined by two long straight sections. The beam is bent around the loops by an array of $\sim 160$ alternating-gradient magnets. The $30^{\circ}$ reverse bends are used to comect the loops to the straight sections. The reverse bencl sistems consist of an array of quadrupoles and bending mogne's which make it possible to adjust the isochronism of son-

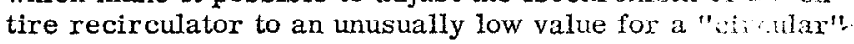
machine. The beam lines for the two long straight, ...ins are located just above the linear accelerator tube in the existing housing.

In the course of one trip around the recirculator, the stored electrons radiate away some of their energy as synchrotron radiation. For example, a $20-\mathrm{GeV}$ electron radiates away about $200 \mathrm{MeV}$ in one turn. The radiation process has several important consequences for the design of the recirculator. For one thing, a high-voltage radiofrequency accelerating system must be provided to restore this lost energy and, for another, the radiation is deposited in a vertically narrow band in the vacuum chambur causing both heating and outgassing which must be specially denlt with. The radiation is of course quantized and thus subject to fluctuations which tend to blow up the bunches of the heam in both the transverse and longitudinal dimensions. This lendency is opposed by radiation damping which can be achieved in all three degrees of freedom by appropriately designing the lattice.

The chief requirement on the lattice of the RLA is that the distribution of particles in 6-dimensional phase space should not grow to be greater than the acceptance of the linear accelerator during the storage interval of $1 / 360$ th of a second. The acceptance of the accelerator is limited by the requirement that a low-energy beam must be accelerated through the same structure. If the high-energy and the low-energy beams are to be accelerated together, which is a requirement for $360 \mathrm{pps}$ operation of the RLA, then the acceptance for the high-energy beam is limited by the strength of the focusing system that will transport the low-enexgy beam. Extra focusing for the high-energy beam alone, in the form of pulsed quadrupole lenses, could be provided, but at the cost of limiting RLA operation to 180 pps. The present focusing system for the accelerator has a matched acceptance in both the horizontal plane and the vertical plane of about $A=0.3 \times 10^{-6} \pi$ meter-radians at $17.5 \mathrm{GeV}$. This can be increased by adding closer spaced quadrupole lenses, or by idding pulsed focusing, or both up to an esimated $\mathrm{A}=0.6 \times 10^{-6} \pi$ radians.

The longitudinal acceptance of the linear accelerator (the acceptance in phase and energy spread) is limited by the requirement that the final high-energy beam have a sufficiently narrow energy spread for efficient experimental use after its second traversal of the linac. For example, a range of $\pm 8^{\circ}$ in phase about the RF crest results in about $1 \%$ spread in energy gained on the second pass, which corresponds to a spread of about $0.5 \%$ in the final spectrum if the energy is doubled, neglecting the energy spread of the stored beam.

The operation of RLA is probably best understood by tracing the history of a bunch of electrons through the system and out to a target. The bunch is injected near the west end of the two-mile accelerator where it joins a bunch of highenergy electrons from the previous pulse which is just 
beginning its second and final trip down the accelerator. Consider the case in which the linear accelerator is capable of $25 \mathrm{GeV}$ total acceleration from end to end, but that injection talses place partway down the accelerator so that the first-acceleration output energy is $17.5 \mathrm{GeV}$, and the recirculator is set to that energy. The two combined beams reach the end of the accelerator with electrons of $42.5 \mathrm{GeV}$ and $17.5 \mathrm{GeV}$. By a suitable magnetic beam transport system, the $42.5-\mathrm{GeV}$ beam is directed into the beam switchyard (BSY) and transported through it to a target. The $17.5-\mathrm{GeV}$ beam, a $1.6 \mu \mathrm{s}$ train of bunches $1 / 3 \mathrm{~ns}$ apart, is bent $12^{\circ}$ southward to the inflection system of the recirculator which includes as its final element a fast kicker magnet to place the trajectory of the incoming beam on the recirculator axis.

The bunches are now recirculating, losing $120 \mathrm{MeV}$ to synchrotron radiation on each turn and gaining a liksc amount from the RF system. While the bunches are travelling around the recirculator, they will pass a low extraction system which is capable of peeling out a small fraction of the electrons on each trun, removing them from the recirculator and directing them into the BSY for experimental use. As mentioned above, this process can increase the duty cycle by a factor of more than 100 , namely to $7 \%$. The longduty-cycle-beam energy is limited, of course, to the maximum storage energy which will be somewhat lower than $20 \mathrm{GeV}$.

For achieving the highest possible energies with the same duty cycle as that of the present linac, the bunches are not peeled out (i.e, the slow extraction system is turned off), but they are retained for $1 / 360$ th of a second, after which time another fast kicker deflects them into an extraction channel which removes them from the recirculator and reinserts them into the west end of the two-mile accelerator for their final acceleration to $42.5 \mathrm{GeV}$.

\section{Main Bend Cells}

The main bend magnets so dominate the radiationinduced beam properties of RLA, that the requirement to maintain a small, tightly-bunched beam virtually dictates the main-bend magnet parameters. Thus, in order to achieve horizontal damping, the horizontal antidamping effect from the focusing magnets must be more than offset by the damping effect from the defocusing magnets. This requirement, together with practical limits on magnet length, gradient and aperture has led to the design of cells with parameters given in Table $i$. An alternating gradient cell is

Table I

Main Bend Cell Parameters

$$
(\mathrm{E}=20 \mathrm{GeV})
$$

Bend Angle of Cell $(\theta)$

Length of Cell ( $(\ell)$

Effective Bend Radius $(\ell / \theta)$

Phase Advance pei. Cell (horizontal)

Phase Advance per Cell (vertical)

Defocus Magnet Bend Angle

Focus Magnet Bend Angle

Defocus Magnet Guide Field

Focus Magnet Guide Field

Defocus Magnet Gradient

Focus Magnet Gradient

$5^{\circ}$
8.29 meters
95.0 meters
$133^{\circ}$
$36.4^{\circ}$
$3.75^{\circ}$
$1.25^{\circ}$
$8.4 \mathrm{kG}$
$5.8 \mathrm{kG}$
$-.58 \mathrm{kG} / \mathrm{cm}$
$1.66 \mathrm{kG} / \mathrm{cm}$

chosen to achleve the largest bending radii possible fand thus the highest beam energy) within the space available. Since the quantum fluctuations drive primarily the horizontal betatron oscillations, exceptionally strong focusing is used in the horizontal plane as evidenced by the large horizontal phase advance per cell. For the same reason, it is possible to allow the vertical focusing to be comparatively weak, thus relieving the requirements for high gradients somewhat.

\section{Reverse Bends}

From Table I it can be seen that the guide field in the defocus magnet is substantially greater than that in the focus magnet. This disparity, which is required to provide horizontal damping, results in somewhat higher total synchrotron radiation loss than that incurred with an isomagnetic guide field, which must be made up by a larger RF system. To achieve sufficient horizontal damping, the disparity would need to be even greater if it were not for a substantial additional contribution to the horizontal damping resulting from the use of gradient magnets together with quadrupoles in the reverse bends. In addition to returning the beam to the main accelerator housing and adding to the horizontal damping, the reverse bends also provide the means of reducing the longitudinal momentum dilation of the bunches. Expressed in terms of the momentum dilation parameter, $\alpha=(\delta \ell / \mathrm{L}) /(\delta \mathrm{E} / \mathrm{E})$, the reverse bends allow operation in the range of $0<\alpha<10^{-4}$. Values of the alpha in this range are necessary if the recirculating electron bunches are to remain in a phase range of $\Delta \phi= \pm 8^{\circ}$.

Because the strength of the reverse bend magnets does not noticably affect the length of the housing, as do the strengths of the main bend cell magnets, the reverse bend magnets are operated with a guide field of approximately half that of the defocus magnets of the main bends. The resulting parameters for the reverse bends are shown in Table II. A schematic layout of the reve; se bends is shown in Fig. 2.

\section{Table II}

Reverse Bend Parameters $(E=20 \mathrm{GeV})$

$\begin{array}{lc}\text { Number of Systems } & 2 \\ \text { Total Bend Angle } & 30^{\circ} \\ \text { Bend Angle per Magnet } & 2.5^{\circ} \\ \text { Magnet Guide Field } & 3.9 \mathrm{kG} \\ \text { Gradient } & 0.29 \mathrm{kG} / \mathrm{cm}\end{array}$

\section{Beam Parameters}

Typical operation at a setting of $\alpha=5 \times 10^{-5}$ for the momentum dilation results in the expected parameters as shown in Table III for $20 \mathrm{GeV}$.

Table III

Typical System Parameters $(\mathrm{E}=20 \mathrm{GeV}$ )

\begin{tabular}{lc} 
Momentum Dilation & $5 \times 10^{-5}$ \\
Length & $6962.8 \mathrm{~meters}$ \\
Radiation Loss & $206 \mathrm{MeV} /$ turn \\
Rotation Frequency & $43 \mathrm{kHz}$ \\
Harmonic Number & 66312 \\
Longitudinal Damping Period & $2.5 \mathrm{~ms}$ \\
Vertical Damping Period & $4.5 \mathrm{~ms}$ \\
Horizontal Damping Period & $3.8 \mathrm{~ms}$ \\
Storage Time & $2.8 \mathrm{~ms}$ \\
Storage Time in Revolutions & 120 \\
Emittance at Reinsertion & $1.3 \times 10^{-7} \pi$ meter-rad \\
Fraction Lost on Reacceleration & $10.7 \%$ \\
\multicolumn{1}{l}{ (Assuming acceptance $3 \times 10^{-6} \pi$ meter-rad) }
\end{tabular}

The key parameter is the fractional beam loss on reacceleration. Even though the emittance at the moment of reinsertion is less than one-half the maximum acceptance of the accelerator, 10.7\% of the beam lies ir "Gaussian tails" beyond the cutoff of the accelerator focusing system and 
aperture. Any mismatching or mis-steering of the beam will add to this loss. Although $10 \%$ is deemed an acceptable beam loss, provided the location of the loss is controlled, modifications to the focusing system are still being studied with the aim of further reducing the loss. Since the loss would increase at higher energy, it would probably be necessary to adopt the technique of pulsed focusing described earlier with the resulting limitation to 180 pps operation.

\section{RF System}

The $R F$ accelerator system is located in sectors 21 and 22 of the main accelerator housing. Sixteen klystrons and accelerator structures (each structure is a standard SLACtype "girder" $40 \mathrm{ft}$ in length) are planned for RLA. The girders are staggered between the east-bound and the westbound beam lines, alternating with $40 \mathrm{ft}$ drift spaces. These spaces are used to locate quadrupole focusing elements and bcam monitoring deviccs. The two sectors ( 640 feet) of accelerating structure are capable of providing a peak accelerating voltage of $230 \mathrm{MV}$.

The klystrons powering the $R F$ system produce peak and average powers of $500 \mathrm{~kW}$ and $55 \mathrm{~kW}$ respectively. These tubes, which are located in the klystron gallery, feed RF power to the accelerating structure in the tunnel below through a waveguide system which is very similar to that used for the present SLAC machine. The tubes are driven by a signal tapped off from the existing SLAC drive line, again adapting many components and techniques developed for the present accelerator. Prototypes of the klystron and modulator operating at the RLA RF pulse length and repetition rate $(2.6 \mu \mathrm{s}, 43,100 \mathrm{pps})$ and capable of reaching a peak output power of $220 \mathrm{~kW}$, have already been successfully tested.

\section{Longitudinal Parameters}

To achieve an adequate quantum lifetime and a sufficiently short bunch length, the bunch must be accelerated at about $25^{\circ}$ to $30^{\circ}$ off the crest of the RF wave (a synchronous phase angle of $115^{\circ}$ to $120^{\circ}$ ). Typical operation of the RF system and the resulting longitudinal parameters are shown in Table $\mathrm{IV}$.

\section{Table IV}

Longitudinal Parameters $(E=20 \mathrm{GeV})$

\author{
Peak RF Voltage \\ RF Phase (from crest) \\ Synchrotron Period (revolutions) \\ Energy "Bucket" $6^{\circ} \mathrm{E} / \mathrm{E} \max$ \\ Energy Spread at Reinsertion \\ Bunch Length at Reinsertion \\ Quantum Lifetime \\ Final $\delta \mathrm{E} / \mathrm{E}$ after Reacceleration
}

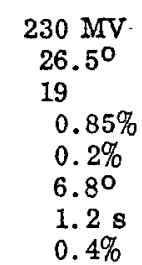

The above parameters are given for recirculation at maximum energy and negligible current; under these conditions nearly all of the RF power is dissipated in the walls of the accelerating structure to establish the energy gradient. At lower recirculation energies, RF power is available to accelerate higher currents. Figure 3 shows the beam loading curve for the proposed RF system capable of a maximum of $35 \mathrm{~mA}$ at $17.5 \mathrm{GeV}$ recirculating energy. For currents of this order, the induced beam voltage becomes a significant fraction of the generator-produced voltage. At a peak current of $35 \mathrm{~mA}$, for example, the beam induced voltage is $83 \mathrm{MV}$. Under conditions of high beam loading, steps must be taken to compensate for the large transient variation in net accelerating voltage, which would take place over about half of the available pulse length. By making appropriate shifts in the phase of the RF power during the RF pulse period, adequate compensation can be made for transient beam loading.

\section{Extraction System}

A large measure of the effectiveness of SLAC has resulted from the multiple beam capability; up to six different beams (plus the SPEAR injection beam) may be interspersed on a pulse-to-pulse basis. The beam extraction system of RIA, as shown in Fig. 4, is intended to preserve this capability. The high-energy beam is scparated from the lowenergy beam at the $0.5^{\circ}$ pulse magnet at the end of the accelerator. The $1^{\circ}$ septum magnet deflects the inflection beam into the recirculating system. 'ithe high-energy beam, which misses the septum, can be directed to any of the three lines in the beam switchyard (BSY). Low-energy beams can be interspersed with the high-energy beams on a pulse-to-pulse basis. In addition, the long-spill line is brought into the BSY from the recirculator by a separate beam transport system. Substantial savings can be made if each pulse magnet group consists of a mixture of dc magnets and pulse magnets capable of pulsing in either direction. In this case, the SPEAR beam is also brought into the BSY by a separate transport system.

\section{Concluding Remarks}

The foregoing has summarized the status of the beam dynamics calculations for the RLA proposal. The cost analysis studies that are necessary for the proposal have been supported by engineering work that is too extensive to be reported in detail in this report. In addition to the klystron development work discussed above, studies of magnet systems and vacuum systems have been conducted with appropriate hardware models. Studies of gas desorption from synchrotron radiation are being made using data from SPEAR. The preliminary design of the instrumentation and control system includes detailed studies of the phase and drive system, comparative studies of different types of beam position monitors and construction of a prototype beam position monitor. Alignment tolerance calculations have been used to determine the number and location of steering magnets. Prellminary designs have been made for all the necessary civil engineering facilities and housings. By the end of the current fiscal year, it is expected that these various studies will be ready for detailed engineering design.

\section{Acknowledgements}

Special mention is due Matt Sands for numerous discussions and contributions to the stability calculations. Richard Helm has devised numerous refinements of the lattice including the reverse bends. Al Lisin and Perry Wilson have directed the mechanical engineering and $R F$ studies, respectively. Dick Scholl and Dave Coward represent the arcas of $\mathrm{I} \& \mathrm{C}$ and beam extraction on the task force. Major contributions to the program have been made by Robert Gould, Martin Lee, Greg Loew, Ken Mallory, Robert Bell, Larry Brown, Willys Brunk, Harry Hoge, William DaviesWhite, Kimo Welch, AI Keicher, and Edward Taylor. Many thanks to those who helped support this effort, and to the leadership, Task Force Leader John Rees and Technical Division Director Richard Neal. 


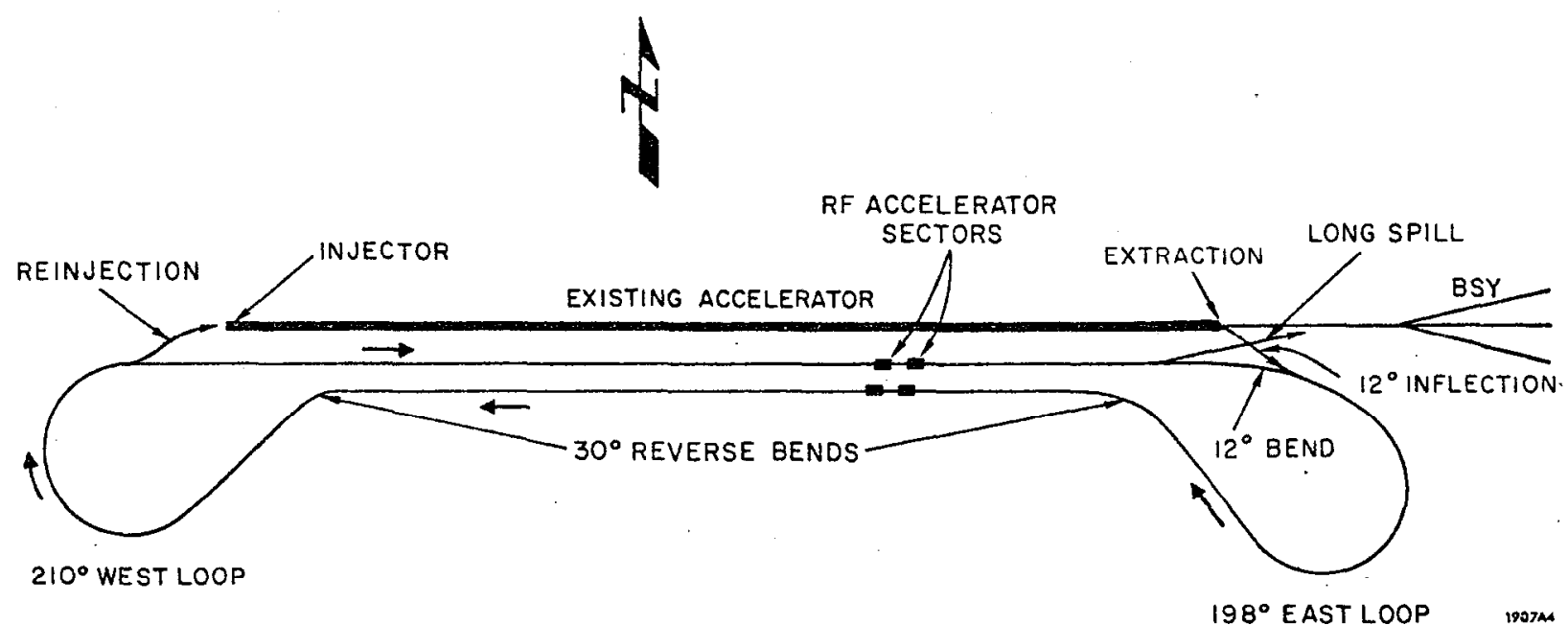

FIG. 1--Proposed layout of RLA.

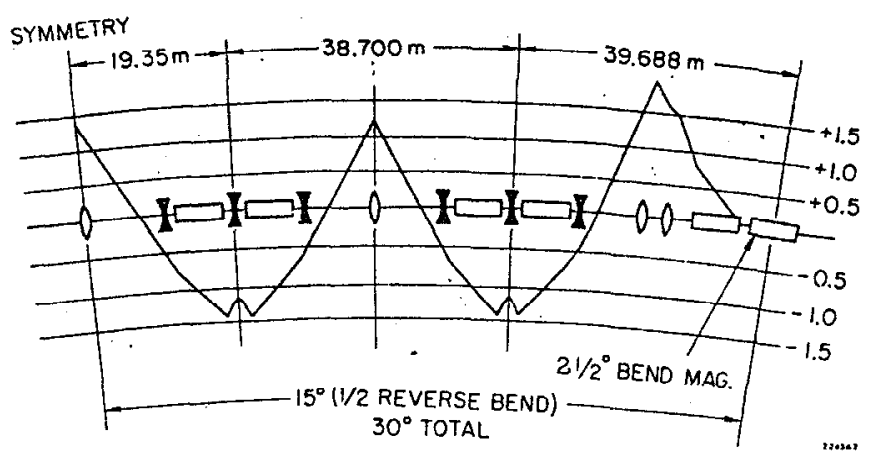

FIG. 2--Reverse bend system showing the function $x(\delta p / p)$.

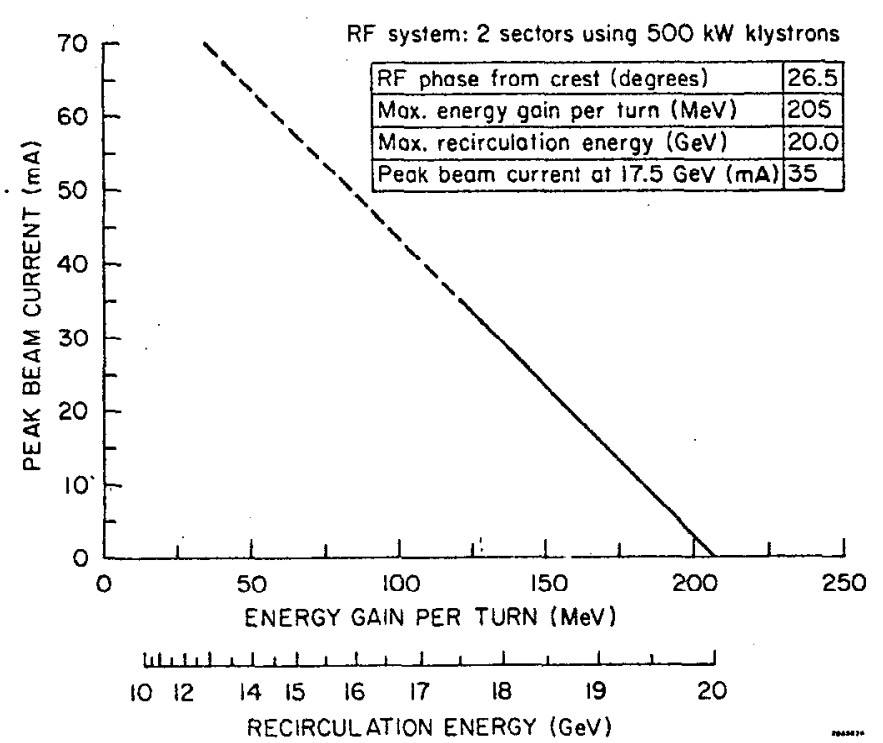

FIG. 3--Load-line diagram for the RLA RF system.

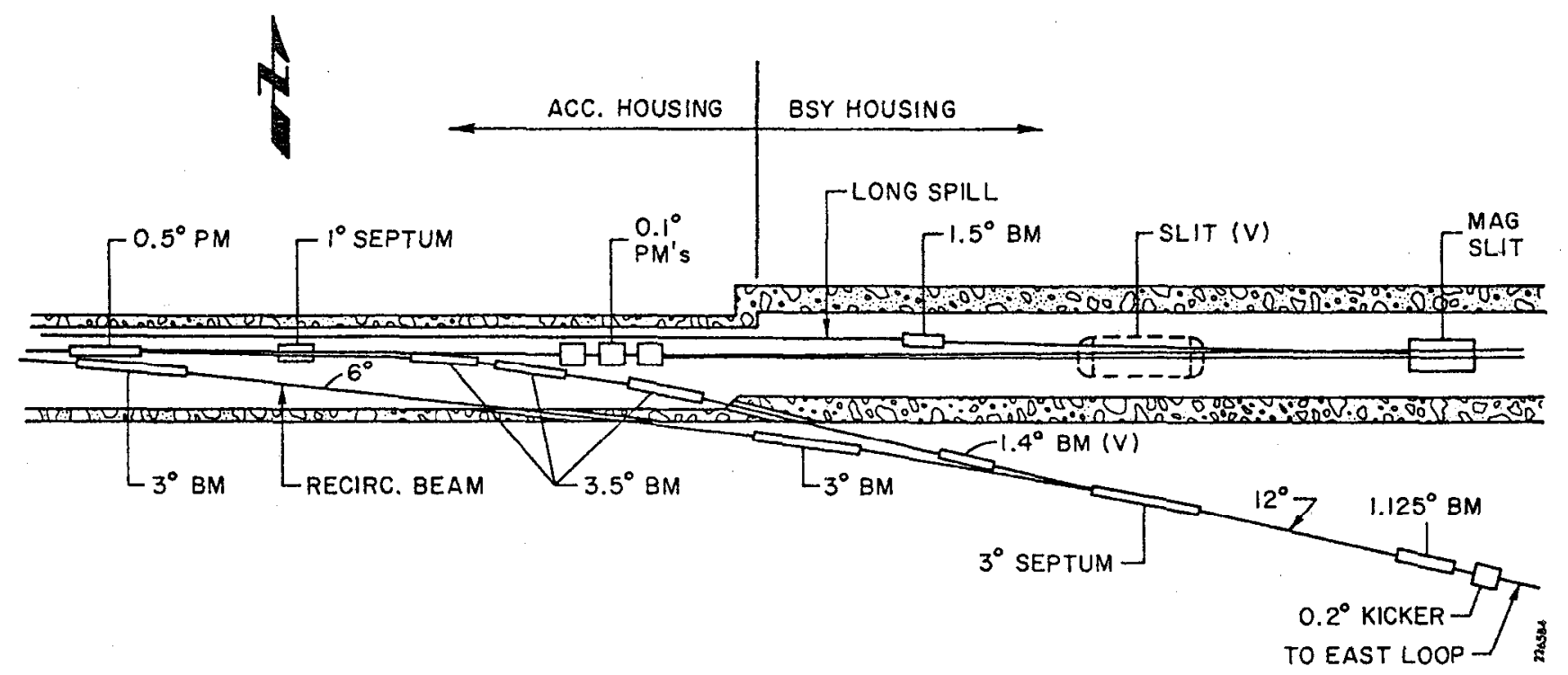

FIG. 4--Beam transfer area at the beginning of the beam switchyard. 